\title{
Vibration analysis for underwater explosion in a small pond with diameter of $\mathbf{5 . 5}$ meters
}

\author{
Quan Wang ${ }^{1,2, a}$,Zhimin Li ${ }^{1, b}$, Youfu Tang ${ }^{1, c}$ \\ ${ }^{1}$ School of Chemical Engineering, Anhui University of Science and Technology, Huainan 232001, \\ China \\ ${ }^{2}$ Postdoctoral Mobile Research Station for Civil Engineering , Anhui University of Science and \\ Technology, Huainan 232001, China \\ awqaust@163.com , bzhmli@aust.edu.cn, c184682601@qq.com
}

\begin{abstract}
Keywords: underwater explosion; vibration signal; Hilbert Huang transform ; explosion mechanics Abstract With the development of marine resources and the demand of marine military protection, underwater explosion technology is getting more and more attention. The underwater explosion experiments with single charge $\&$ double charges were carried out in a small cylinder explosion pond with diameter of $5.5 \mathrm{~m}$, height of $3.62 \mathrm{~m}$ in the paper. Blasting vibration testers of Ubox-5016 type were adopted to detect the vibration signal near the pond, and the results show that: (1) With the increasing of distance, the vibration velocity decreases, while the main vibration frequency increases. And, the increasing of the charge quantity, the vibration velocity increases. (2) From Hilbert energy spectrum analysis, the frequency distribution of underwater explosion vibration energy is within the range of $100 \mathrm{~Hz}$, and the main energy is mainly concentrated in low frequency region below $50 \mathrm{~Hz}$, but, the energy components for the frequency band range from 50 to $100 \mathrm{~Hz}$ is less. Moreover, the marginal spectrum analysis shows that the main vibration frequency band is in the range of 3 to $10 \mathrm{~Hz}$. (3)Through the analysis of the energy spectrum and the marginal spectrum of the vibration signals by the HHT transform. It further verifies that the millisecond delay initiation can reduce the vibration energy of underwater explosion and the vibration effects. The results and analysis in the paper have important reference value for the design of hydraulic structures and the protection of underwater military confrontation.
\end{abstract}

\section{Introduction}

The seismic wave caused by underwater explosion transient non-stationary signal without fixed frequency. The traditional Fourier analysis is a function defined on the global. It is often not applicable for instantaneous frequency[1]. We have several main analysis methods[2] currently for non-stationary signals, such as Continuous Wavelet Transform, Multi-resolution Analysis, Discrete Wavelet Transform, Wavelet Packet Transform and Hilbert-Huang Transform which is based on Hilbert Transform and proposed by Huang NE et al[3,4]. Penga Z K[5] improved Hilbert Huang Transform (i.e. HHT) and applied to the vibration signal analysis. The HHT analysis method has been successfully applied to the chamber blasting and blasting vibration signal analysis of Open Pit Mine by Li Xibing and Zhang Yiping[3,6]. The results showed that the HHT method is feasible, reliability in blasting vibration signal analysis. By comparing different wavelet analysis methods, Qian Shouyi[7] proved the feasibility of HHT method when applied to the identification of the actual millisecond blasting vibration signal delay time. Gong $\min [8]$ proved EMD identification method is suitable for a small interval millisecond blasting through HHT analysis of engineering example. Time-energy density method and time-frequency domain transform techniques are applied to millisecond blasting by Shi Linghua[9] et al. Jia hu[10] used HHT method to extract underwater explosion pressure signal of metal detonating cord, the experiment was carried out in $\phi 5 m \times 5 m$ underwater explosion test pond. The results showed that HHT method is suitable for pressure signal analysis of underwater explosion. $\mathrm{Xu}$ Zhenyang[11] collected vibration signal of electronic initiation, and used EMD algorithm to analyze the law of vibration energy distribution, and introduced it into the safety protection of the building. The underwater millisecond delay explosion experiments with RDX single charge \& RDX 
double charges were carried out in a small cylinder explosion pond. Ubox-5016 type of blasting vibration testers were used to acquire the vibration signals which was for further FFT analysis. We take HHT method into the field of underwater explosion vibration signal analysis and investigate the effects of millisecond delay initiation on underwater explosion vibration signal. By comparing the obtained energy spectra and marginal spectrum, we have further validate that the millisecond delay initiation can reduce underwater explosion vibration energy. The analysis and experimental results of this paper could provide a theoretical reference for research on underwater engineering blasting, seismic design of hydraulic structures and underwater military confrontation.

\section{HHT Analysis Method}

HHT (Hilbert Huang Transform) analysis method is suitable for processing nonlinear and non-stationary signal. It mainly consists of Empirical Mode Decomposition (EMD) and Hilbert transform[2,4]. Compared with the wavelet analysis, EMD overcomes the problem of wavelet base selection, and avoid the effects due to different base functions. In addition, we get intrinsic mode function IMF which has the characteristics of different scales after processing the original signal with EMD decomposition characteristics obtained with different scales, this process is adaptive and efficient. Then, processing the IMF components with Hilbert transform, we obtained instantaneous spectrum of each IMF component. Hilbert spectrum was obtained by integrating instantaneous spectrum of all IMF components.

The original signal caused by explosion vibration contains signals in three directions, Vertical vibration signal $X(t)$ were selected to analysis in accordance with the provisions of article 13.2.2 of the "Blasting Safety Regulations" (GB6722-2014 in China).

Then, EMD decomposition and HHT transform should be explained. It can be expressed as the following equation after $\mathrm{n}$ times' EMD decomposition of the original signal $X(t)$ :

$$
\mathrm{X}(\mathrm{t})=\sum_{i=1}^{n} C_{i}(t)+r_{n}(t)
$$

Where $C_{i}(t)$ is the $\mathrm{i}$-th component of the IMF by EMD decomposition, $r_{n}(t)$ is residual error that is the original signal minus all IMF components. The obtained IMF component by Hilbert transform is expressed as:

$$
\mathrm{H}[C(t)]=\frac{1}{\pi} P V \int_{-\infty}^{\infty} \frac{C\left(t^{\prime}\right)}{t-t^{\prime}} d t^{\prime}
$$

Construct analytic signal $Z(t)$ :

$$
\mathrm{Z}(\mathrm{t})=\mathrm{C}(\mathrm{t})+\mathrm{jH}[\mathrm{C}(\mathrm{t})]=\mathrm{a}(\mathrm{t}) \mathrm{e}^{\mathrm{j} \varphi(\mathrm{t})}
$$

Where $a(t)$ is the amplitude function.

$$
\mathrm{a}(t)=\sqrt{C^{2}(t)+H^{2}[C(t)]}
$$

$\phi(t)$ is the phase function:

$$
\phi(t)=\tan ^{-1} \frac{H[C(t)]}{C(t)}
$$

The decomposed original signal can be expressed as:

$$
\mathrm{X}(t)=\operatorname{Re} \sum_{\mathrm{i}=1}^{\mathrm{n}} \mathrm{a}_{\mathrm{i}}(t) \mathrm{e}^{\mathrm{j} \Phi_{\mathrm{i}}(t)}=\operatorname{Re} \sum_{\mathrm{i}=1}^{\mathrm{n}} \mathrm{a}_{\mathrm{i}}(t) \mathrm{e}^{\int \Phi_{\mathrm{i}}(\mathrm{t}) \mathrm{dt}}
$$

Hilbert spectrum and marginal spectrum can be expressed as formula (7) and (8), respectively:

$$
\begin{aligned}
& H(w, t)=\operatorname{Re} \sum_{\mathrm{i}=1}^{\mathrm{n}} \mathrm{a}_{\mathrm{i}}(\mathrm{t}) \mathrm{e}^{\int \bar{\omega}_{\mathrm{i}}(\mathrm{t}) d t} \\
& h(w)=\int_{0}^{T} H(w, t) d t
\end{aligned}
$$


Based on the above theory, the author compiled the corresponding code in MATLAB and load corresponding module to make the relevant spectrum.

\section{Underwater Explosion Vibration Test in a Small Pond}

The test was carried out in a small cylinder explosion pond with diameter of $5.5 \mathrm{~m}$, height of 3.62 $\mathrm{m}$. The author made phlegmatized-RDX into spherical waterproof charge, initiated with $8 \#$ industrial detonator, the charge interval is $30 \mathrm{~cm}$. The three charges were $5 \mathrm{~g}$ of single charge, $10 \mathrm{~g}$ of single charge and $5 \mathrm{~g}+5 \mathrm{~g}$ of double-charge. The distance from water of charges are $2.4 \mathrm{~m}$ during the test. Ubox-5016 type of blasting vibration tester was used to acquire the signal. Sensors are arranged $1 \mathrm{~m}$ and $2 \mathrm{~m}$ from the outer surface of the cylinder pond (blast center distance is 3.75 and $4.75 \mathrm{~m}$ ).

Fixed mount

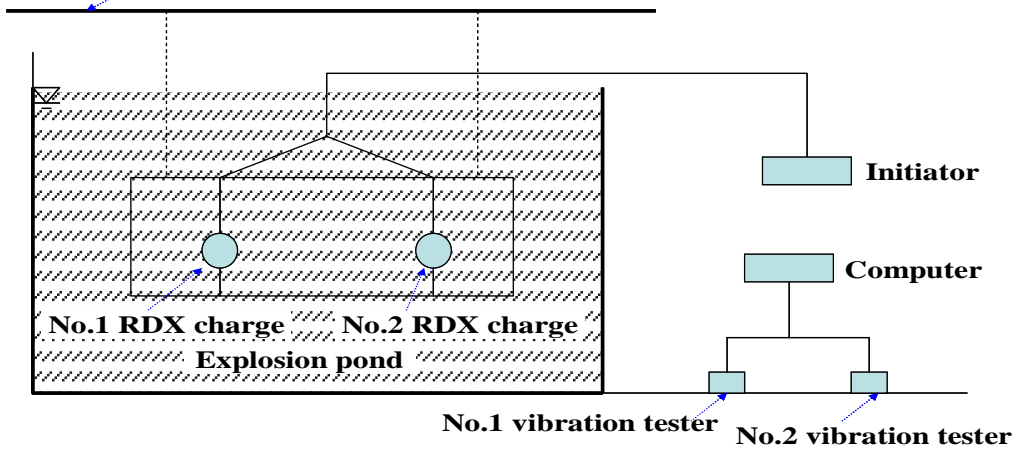

Fig.1 Experimental system of underwater explosion

\section{Test results of explosion vibration}

Table 1 the measured data of explosion vibration

\begin{tabular}{clccc}
\hline Distance & \multicolumn{1}{c}{ Parameters for vibration signals } & 1\# / 4\# & 2\# / 5\# & 3\# / 6\# \\
\hline Main vibration frequency/Hz & $4.88 / 61.04$ & $9.77 / 61.04$ & $6.10 / 26.86$ \\
& Maximum velocity of vibration /cm/s & $0.055 / 0.079$ & $0.061 / 0.10$ & $0.039 / 0.067$ \\
$\mathrm{~T}$ & Time of the maximum velocity /ms & $18.80 / 134.20$ & $125.60 / 20.40$ & $105.20 / 19.40$ \\
& Vibration duration/s & $0.95 / 0.95$ & $0.95 / 0.95$ & $0.95 / 0.95$ \\
& Main vibration frequency/Hz & $61.04 / 4.88$ & $61.04 / 9.77$ & $26.86 / 6.10$ \\
$1 \mathrm{~m}$ & Maximum velocity of vibration /cm/s & $0.081 / 0.038$ & $0.10 / 0.060$ & $0.075 / 0.038$ \\
$\mathrm{R}$ & Time of the maximum velocity /ms & $15.40 / 81.60$ & $18.00 / 120.60$ & $17.20 / 104.20$ \\
& Vibration duration/s & $0.95 / 0.95$ & $0.95 / 0.95$ & $0.95 / 0.95$ \\
& Main vibration frequency/Hz & $4.88 / 28.08$ & $9.77 / 9.77$ & $4.88 / 26.86$ \\
Maximum velocity of vibration /cm/s & $0.46 / 0.44$ & $0.73 / 0.65$ & $0.54 / 0.46$ \\
V Time of the maximum velocity /ms & $24.40 / 140.40$ & $40.00 / 43.00$ & $26.00 / 24.80$ \\
Vibration duration/s & $0.94 / 0.93$ & $0.92 / 0.94$ & $0.94 / 0.94$ \\
\hline
\end{tabular}

Note: T: tangential, R: radial, V: vertical

(1) $1 \#$ or $4 \#$ is $5 \mathrm{~g}$ RDX of single charge + single detonator (TNT-equivalent $7.46 \mathrm{~g}$ )

(2) $2 \#$ or $5 \#$ is $10 \mathrm{~g}$ RDX of single charge + single detonator (TNT-equivalent $13.84 \mathrm{~g}$ )

(3) $3 \#$ or $6 \#$ is $5 \mathrm{~g}$ RDX of double charge + double detonator (TNT-equivalent $14.92 \mathrm{~g}$ )

The experimental results of underwater explosion are as follows:

(1) According to Table 1, compare the maximum velocity of three directions (tangential, radial and vertical), We will find the maximum vertical vibration velocity is much greater than the maximum tangential vibration velocity and maximum radial vibration velocity. According to provisions of article 13.2.2 of the "Blasting Safety Regulations" (GB6722-2014), we will take the vertical component for further analysis.

(2) The maximum vertical vibration velocity gradually decreases with increasing distance in a fixed amount of charge, the maximum vibration velocity increases with the charge increases in a fixed 
measuring distance.

(3) Compared with test (2), we use MS-3\# and MS-5\# detonators to achieve millisecond delay initiation in the test (3). It is obvious that the maximum velocity of delay initiation will be reduced by about $30 \%$ in a same distance $(1$ or $2 \mathrm{~m}$ ) from Table 1 , but there is no significant change for main vibration frequency. It can be seen that segmented time-delay underwater explosion is less harmful to the environment, millisecond delay blasting can properly control the maximum vibration velocity and reduce the harm of blasting vibration in engineering practice.

\section{HHT analysis of explosion vibration signals}

According to the vibration signal collected by blasting vibration testers, vibration signals of $10 \mathrm{~g}$ RDX of single charge and $5 \mathrm{~g}$ RDX of double charge (use MS-3\# and MS-5\# detonators to achieve millisecond delay initiation) in vertical direction at $1 \mathrm{~m}$ were dealt with EMD decomposition and reconstruction. Then, we got Hilbert-Huang energy spectrum and marginal spectrum.

EMD decomposition and reconstruction of explosion vibration signals

We take vibration signal of $5 \mathrm{~g}$ RDX of single charge in vertical direction at $1 \mathrm{~m}$ as an example to do EMD decomposition and reconstruction.
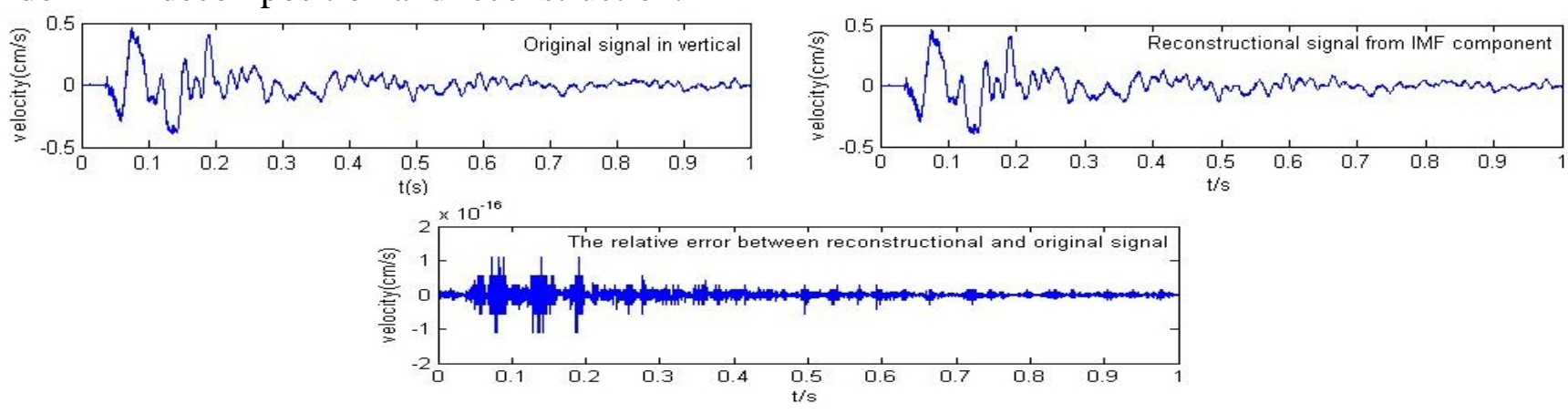

Fig. 2 the original signal, the reconstructed signal and the relative error distribution

From Fig. 2, the relative error between reconstruction signal and original signal is $10^{-16}$, with a high degree of consistency between them, it can accurately reflect the characteristics of original signal and ensure the reliability of the subsequent analysis.
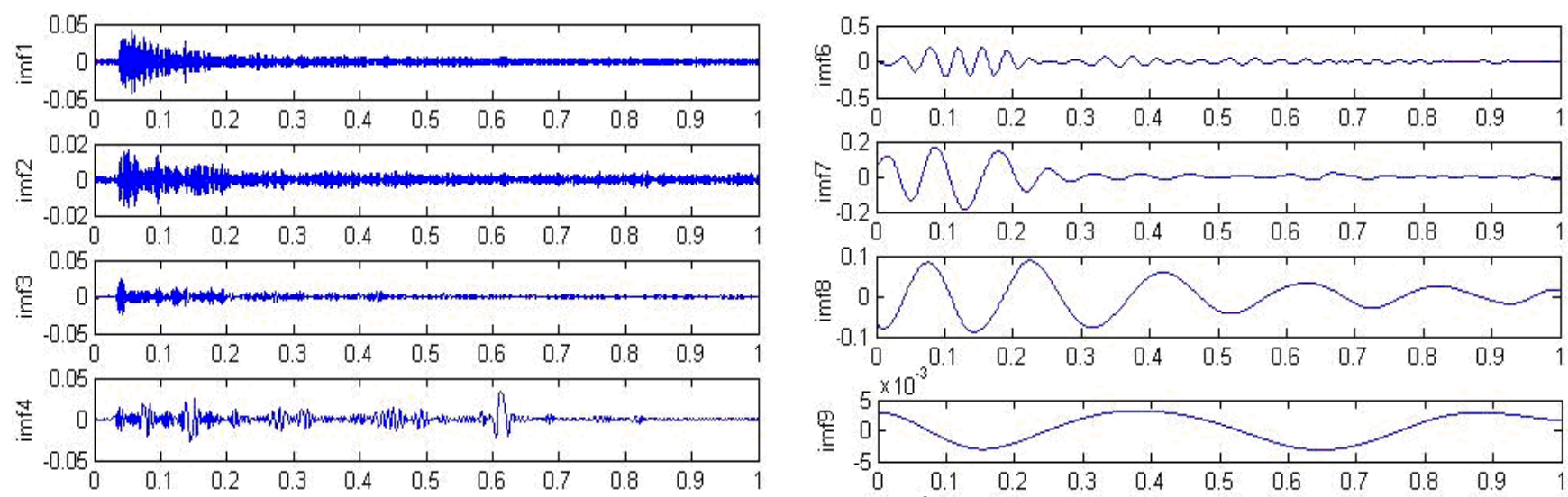

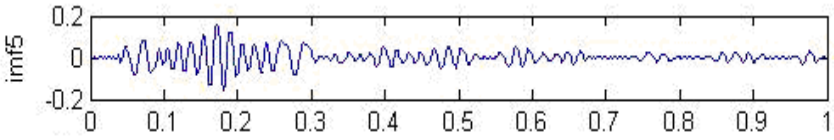

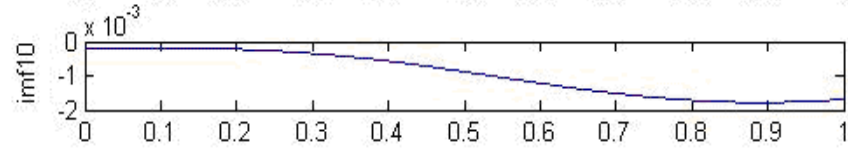

Fig. 3 the IMF component obtained from the EMD decomposition of vibration signal in vertical

We firstly dealt explosion vibration signal in vertical with EMD decomposition from Fig. 2 and then got 9 IMF components and a residue which were shown in Fig. 3. Wherein, component imf 1 got the highest frequency, but content low energy, indicating that the signal contained white noise; component imf 2 represents the high-frequency parts of the signal; imf 1 - 6 got the largest amplitude and contained more energy, it was the dominant frequency segment of the signal, comprised the most significant characteristics of the signal; component imf 7 - 9 represent the low-frequency parts of the signal; amplitude of residue was small, it may be weak signal or wandering of instrument. 
The process of EMD decomposition has characteristics of high efficiency and adaptability, to ensure that the decomposed signal remains characteristics of non-stationary of underwater explosion vibration signals. It also indicates that HHT analysis method is suitable for underwater explosions vibration signal analysis.

\section{Analysis on Hilbert energy spectrum of explosion vibration signals}

Hilbert energy spectrum is each of the IMF components showing in the color-coded graphs in the form of frequency - time - amplitude image (The amount of energy shown on the right side of the color-coded bars, namely: the darker the dot, the larger the energy).

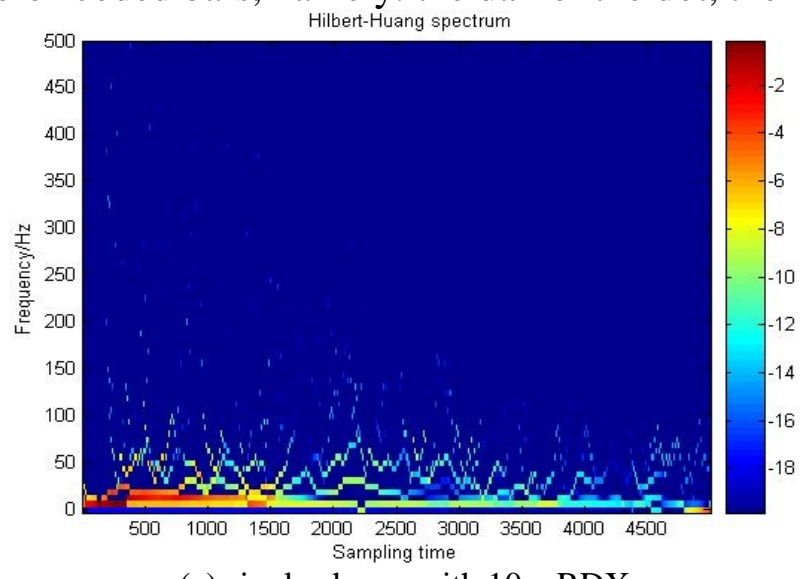

(a) single charge with $10 \mathrm{~g}$ RDX

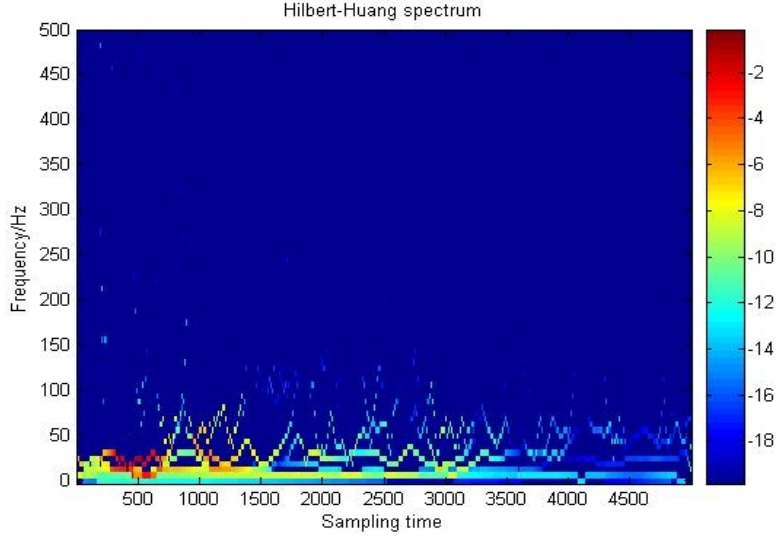

(b) double charges with $5 \mathrm{~g}$ RDX each

Fig.4 Hibert-Huang energy spectrum of explosion vibration signal

It can be seen from Fig. 4(a) and 4(b) that the frequency of underwater explosion vibration energy is less than $100 \mathrm{~Hz}$, and the energy is concentrated in the low frequency region below $50 \mathrm{~Hz}$, less energy distributed in frequency band of $50-100 \mathrm{~Hz}$; there are few energy distribution in higher frequency band above $100 \mathrm{~Hz}$. By comparison, at the same amount of charge, the main range of energy distribution is more concentrated and relatively less vibration energy components when use millisecond delay initiation. This is due to vibration waveform superimposed action. And then weaken the vibration amplitude. By comparing the energy spectrum, we verified the energy of underwater explosion vibration can be reduced by millisecond delay initiation.

\section{Analysis on Hilbert marginal spectrum of explosion vibration signals}

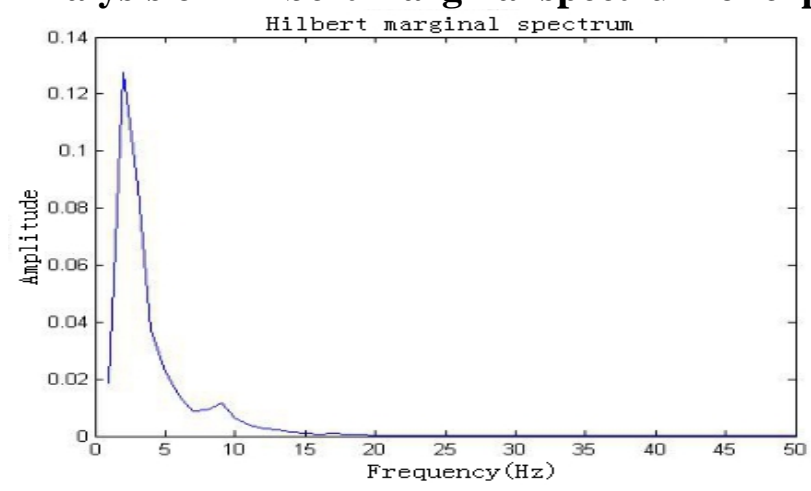

(a) single charge with $10 \mathrm{~g}$ RDX

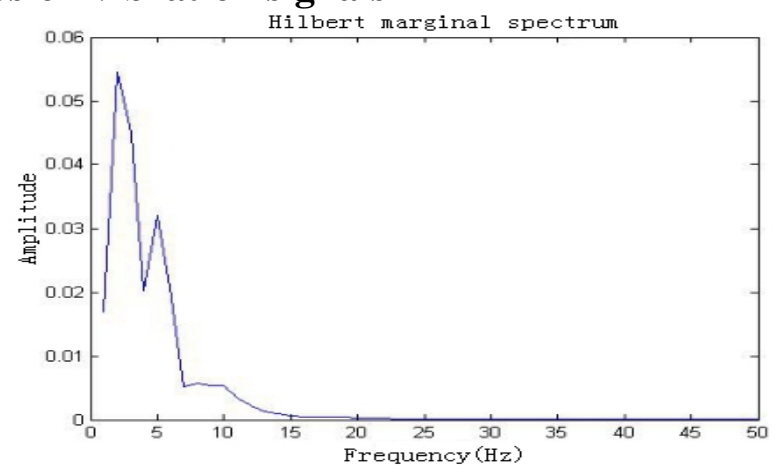

(b) double charges with $5 \mathrm{~g}$ RDX each

Fig.5 the marginal spectrum of explosion vibration signal

The marginal spectrum in Fig. 5(a) and 5(b) were drawn by taking date from tests (2) \& (3) into Equation 8 for calculation. We can draw the following conclusions: It is mainly low-frequency in short distance of underwater explosion vibration with small charge. Its main vibration frequency band of $3-10 \mathrm{~Hz}$.

The dominant frequency band of single charge with instantaneous initiation contains an obvious sub-frequency band in Fig. 5(a). But The dominant frequency band of double charge with millisecond delay initiation contains two obvious sub-frequency bands in Fig. 5(b). The single-peak amplitude was 0.13 when we took single charge with instantaneous initiation, the double-peak amplitude were 0.055 and 0.033 when we took double charge with millisecond delay initiation. 
The main vibration frequency band can be dispersed by millisecond delay initiation, and it can reduce the total energy of the vibration so as to decrease vibration intensity and vibration damage. It further verified that underwater explosion vibration energy can be reduced by millisecond delay initiation. In short, explosion vibration can be well controlled when we use millisecond delay initiation. It also can be applied into engineering practice.

\section{Conclusions}

Based on the above experiment, the following conclusions were drawn:

(1) The vibration velocity gradually decreases with increasing distance in a fixed amount of charge, the vibration velocity increases with the charge increases in a fixed measuring distance.

(2) By Hilbert energy spectrum analysis, it indicate that the frequency of underwater explosion vibration energy is less than $100 \mathrm{~Hz}$, and the energy is concentrated in the low frequency region below $50 \mathrm{~Hz}$, less energy distributed in frequency band of 50-100 Hz. By Hilbert marginal spectrum analysis, it indicate that the main vibration frequency band of 3-10 Hz.

(3) By Hilbert energy spectrum analysis and Hilbert marginal spectrum analysis, it further verified that underwater explosion vibration energy can be reduced by millisecond delay initiation .The analysis and experimental results of this paper could provide a theoretical reference for seismic design of hydraulic structures and underwater military confrontation.

\section{Acknowledgements}

This study was supported by the key project of national natural science research in China (No. 11502001), and the postdoctoral foundation project in China \& Anhui Province (No. 2014M561808, No. 2014B035), the authors expressed their sincere gratitude here!

\section{Reference}

[1] Pai P F. Nonlinear vibration characterization by signal decomposition[J]. Journal of Sound and Vibration, 2007, 307(3): 527-544.

[2] Huang N E, Shen Z, Long S R. A new view of nonlinear water waves: The Hilbert Spectrum[J]. Annual review of fluid mechanics, 1999, 31(1): 417-457.

[3] ZHANG Yi-ping. HHT Analysis of Blasting Vibration and Its Application[D]. Doctor Thesis, Changsha: Middle and Southern University, 2006.

[4] Huang N E, Shen Z, Long S R, et al. The empirical mode decomposition and the Hilbert spectrum for nonlinear and non-stationary time series analysis[C]//Proceedings of the Royal Society of London A: Mathematical, Physical and Engineering Sciences. The Royal Society, 1998, 454(1971): 903-995.

[5] Peng Z K, Peter W T, Chu F L. An improved Hilbert-Huang transform and its application in vibration signal analysis[J]. Journal of sound and vibration, 2005, 286(1): 187-205.

[6] ZHANG Yiping, LI Xibing, ZHAO Guoyan, et al. Analysis of Chamber Blasting Vibration Based on HHT Method[J]. Chinese Journal of Rock Mechanics and Engineering,2006, 37(1): 150-154.

[7] QIAN Shouyi, LI Qiyue. HHT Instantaneous Energy Method to Identify of Delay Time in Millisecond Blasting[J]. Mining Research and Development, 2012, 32(2): 113-116.

[8] GONG Min, QIU Yikeke, MENG Xiangdong, et al. Identification Method of Detonator's Actual Firing Time Delay Based on HHT and Its Application in Millisecond Blasting Under Urban Environment[J].Journal of Vibration and Shock, 2015, 34(10): 206-212.

[9] LING Tonghua, LI Xbing, WANG Guiyao. A Study on Initiative Control of Blast Vibration Damages[J]. Rock and Soil Mechanics, 2007, 28(7): 1439-1442.

[10] JIA Hu, ZHENG Weihua, LUO Qiang. The Characteristics of Time Frequency Signal Based on Hilbert-Huang Transform in Underwater Explosion by Metal Detonating Cord[J]. Engineering Blasting, 2015, 21(3): 5-8. 
[11] XU Zhenyang, YANG Jun, CHEN Zhanyang, et al. Blasting Seismic Waves Energy Distribution Based on EEMD[J]. Journal of Vibration and Shock , 2014, 33(11): 38-42. 